\title{
Surface roughness pattern measurement of tensile specimen by using digital holography
}

\author{
Kyeong-suk,Kim ${ }^{1,}$, , Dong-pyo,Hong, ${ }^{2, b}$, Man-yong,Choi ${ }^{3, c}$, Hyun-chul,Jung ${ }^{4, d}$ \\ Dong-soo, $\mathrm{Kim}^{5, \mathrm{e}}$,Sung-won, $\mathrm{La}^{6, \mathrm{f}}$ and Ho-seob,Chang ${ }^{7, \mathrm{~g}}$ \\ 1,4 Department of Mechanical Design Engineering, Chosun University \\ ${ }^{2}$ Division of Precision Mechanical Engineering, Chonbuk National Univeristy \\ 664-14 1Ga Duckjin-Dong, Duckjin-Gu, Jeonju, Jeonbuk, 561-756, Korea \\ ${ }^{3}$ Korea Research Institute of Standard and Science (KRISS) \\ P.O.Box 102 Yuseon, Daejeon, 305-600, Korea \\ ${ }^{5,6}$ Department of Advanced Parts and Materials Engineering \\ ${ }^{7}$ Laser Center, Chosun University \\ 375 Seoseok-Dong, Dong-Gu, Gwangju, 501-759, Korea
}

a gsckim@chosun.ac.kr, ${ }^{b}$ hongdp@chonbuk.ac.kr, ${ }^{c}$ mychoi@kriss.re.kr, ${ }^{d}$ yonggary@ieee.org,
${ }^{e}$ victoryehdtn@naver.com, ${ }^{f}$ infinite love@nate.com, ${ }^{9}$ hschang@chosun.ac.kr

Keywords: Surface Roughness, Digital holographic interferometer, Tensile Testing Machine

\begin{abstract}
The objective of this paper is measurement of the surface roughness of tensile specimens under different tensile speed. In the test, the tensile specimens were loaded by tensile testing machine. The roughness of the surfaces was measured by digital holographic interferometric system (DHI). From the results, it was confirmed that DHI could measure the roughness of the various types of the specimen surface. In this paper, as the tensile speed was faster, the roughness of specimen surface was smaller.
\end{abstract}

\section{Introduction}

The application of holographic measurement field has developed with the demand of precision measurement since the 1960s, and it was mostly applied in the measurement using interference pattern such as the holographic interferometer [1]. In the digital holography, the laser beam split into two beam, one is incident by penetrating or reflecting it into the CCD camera and the other is incident into the CCD camera as reflecting from the reference mirror. The object wave and the reference wave make the interference fringes which are called "Digital Hologram", the information of object is analyzed by reconstructing the hologram numerically. By applying the optic measurement method which is the digital holography to the precision measurement of the surface roughness, it could have a positive effect on the union and development of the related knowledge. Because of the development of directly fast and precise measurement system, it makes a contribution to the industrial growth of the related application fields. The mechanical application of holography could be increased through this paper. With this propose, the surface characteristic of the specimen were measured quantitatively by stretching the standard tensile specimen with various speeds. [2][3]

\section{Theory}

\subsection{Recoding of Hologram}

The concept of digital hologram recording is illustrated in Fig.1.1. A plane wave reference and the wave reflected from the object are interfering at the surface of a CCD. The resulting hologram is electronically recorded and stored. The object is general a three dimensional body with diffusively reflecting surface, located at a distance $d$ from the CCD.

Total of two intensity which is interfered, 
$I(x, y)=|A(x, y)|^{2}+|a(x, y)|^{2}+2|A(x, y)||a(x, y)| \cos |\Psi(x, y)+\Phi(x, y)|$

Eq.1.1

Where $\mathrm{I}(\mathrm{x}, \mathrm{y}), \mathrm{A}(\mathrm{x}, \mathrm{y}), \mathrm{a}(\mathrm{x}, \mathrm{y}), \Psi(\mathrm{x}, \mathrm{y})$ and $\Phi(\mathrm{x}, \mathrm{y})$ are interfered intensity, reference Beam, object beam, reference beam's phase and object beam's phase respectively.

Eq.1.1 simply comes to be defined,

$$
I(x, y)=|a(x, y)+A(x, y)|^{2}=|a|^{2}+|A|^{2}+a A+a * A
$$

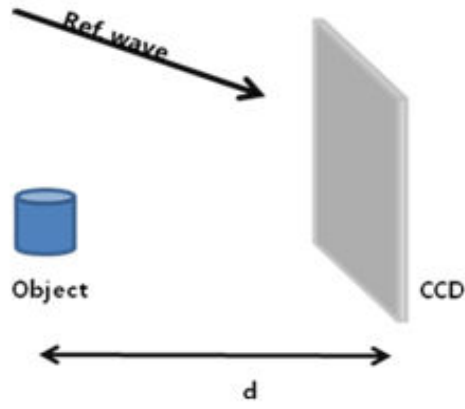

Fig.1.1 Interferometer record

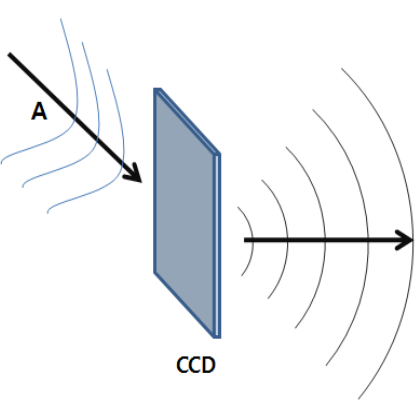

Fig. 1.2 (a) Reference beam

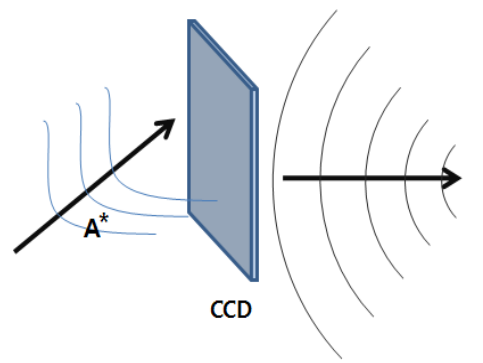

(b) Conjugate reference beam

\subsection{Reconstruct of Hologram}

For holographic reconstruction, the hologram function has to be multiplied with the complex amplitude of the reconstruction (reference) wave;

$$
A(x, y) I(x, y)=\left(|a|^{2}+|A|^{2}\right) A+|A|^{2} a+a *|A|^{2}
$$

The first term on the right side of Eq.1.3 corresponds to the un-diffracted wave passing the hologram (zero diffraction order) in optical reconstruction. The second term is the reconstructed object wave, forming the virtual image. The third term generates a distorted real image of the object. The virtual image appears at the position of the original object and the real image is formed at a distance $d$ as well, but in the opposite direction from the CCD as Fig.1.2 show. The reason for the distortion of the real image of the spatially varying complex factor $\mathrm{A}(\mathrm{x}, \mathrm{y})$, which modulates the image forming conjugate object wave $A^{\prime}(x, y)$. An undistorted real image can be generated by using the conjugate reference beam $\mathbf{A}^{\prime}(\mathrm{x}, \mathrm{y})$ as shows Fig.1.2

$$
A^{\prime \prime}(x, y) I(x, y)=\left(|a|^{2}+|A|^{2}\right) A^{\prime}+A^{\prime 2} \mathrm{a}+\mathrm{a} *|A|^{2}
$$

\subsection{Angular spectrum}

The angular spectrum method, also known as plane wave expansion method, is seen to be fairly efficient computationally but with the highest degree of accuracy. The object field on the hologram plane, $\mathrm{U}(\mathrm{x}, \mathrm{y})=\mathrm{u}(\mathrm{x}, \mathrm{y}) \mathrm{e}^{\mathrm{i} \boldsymbol{\mathrm { I }} \mathrm{x}, \mathrm{y})}$, is expressed as a Fourier integral;

$\mathrm{U}(\mathrm{x}, \mathrm{y})=\int \mathrm{dk} \mathrm{dk}_{\mathrm{y}} \mathrm{A}\left(\mathrm{k}_{\mathrm{x},} \mathrm{k}_{\mathrm{y}}\right) \exp \left[1\left(\mathrm{k}_{\mathrm{x}} \mathrm{x}+\mathrm{k}_{\mathrm{y}} \mathrm{y}\right)\right]$

Its two dimensional Fourier transformation $A\left(k_{x}, k_{y}\right)=F_{n}\left[U\left(x_{y} y\right)\right], F_{n}$; Fourier transform is considered as the amplitude of the wave whose wave vector is Eq.1.6.

$\vec{k}=k_{x} \hat{x}+k_{y} \hat{y}+k_{z} \hat{z}:$ Wave factor

Then, the object field on the reconstruction plane is obtained as $\mathrm{U}(\mathrm{X}, \mathrm{Y}: \mathrm{Z})=\int \mathrm{d} \mathrm{k}_{x} \mathrm{dk}, \mathrm{A}\left(\mathrm{k}_{x}, \mathrm{k}_{y}\right) \exp \left[\mathrm{i}\left(\mathrm{k}_{x} \mathrm{X}+\mathrm{k}_{\mathrm{y}} \mathrm{Y}\right)\right] \exp (\mathrm{ik}, \mathrm{Z})$ 


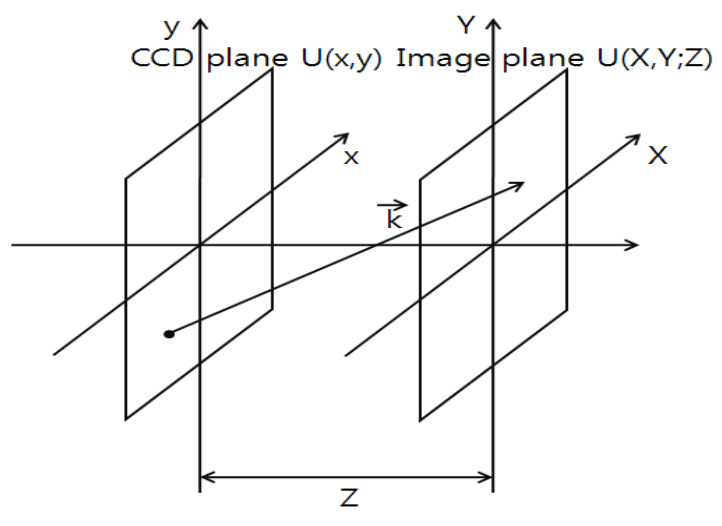

Fig.1.3 Coordinate system for reconstruction

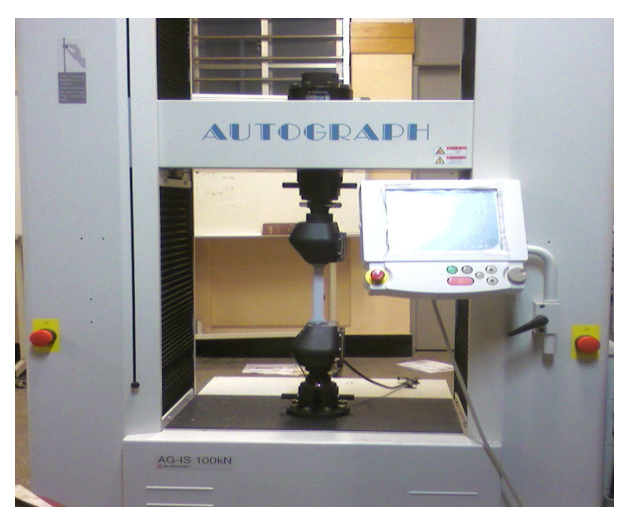

Fig. 2.1 UTM Device

\subsection{Change of height distribution}

After the information recorded in CCD from digital holography interferometer was reconstructed numerically with computer, then the phase information of the object surface are obtained. The phase information of each layer with focusing on its layer must be gathered to measure the precise height. The measured whole phase distribution can convert into the height distribution and the convert equation is shown as Eq.1.8.

$$
\Delta \mathrm{h}=\frac{\Delta \phi}{4 \pi} \cdot \lambda
$$

\section{Experiment setup and method}

\subsection{Experiment equipment}

\subsubsection{Tensile test machine}

The tensile tester uses SHIMADZU products (AG-IS $100 \mathrm{kN}$ ), the composition which equipment is Fig.2.1 is same. The test which follows in tensile tester tension test, compression test and hoof force tentative etc. standard is possible, the test of long period is possible and is equipment where the test of the material which is various is possible.

\subsubsection{Reflective interferometer}

The composition of the interferometer which is used in experiment of the present paper Fig 2.2 is same. The interferometer which sees does not transmit the illuminant not to be able, is a method which uses in the objective which reflects. With the object group the reference group interference occurs from CCD surfaces and has the hologram which comes to be gotten and phase shift presumption and phase treatability leads and finds out shame remaking algorithm leads and remakes the complex amplitude and a phase of the object, group. Also we composed a system for magnification measurement by putting the objective lens in front of the object. According to magnification ratio of the object lens will be the possibility of raising a resolution
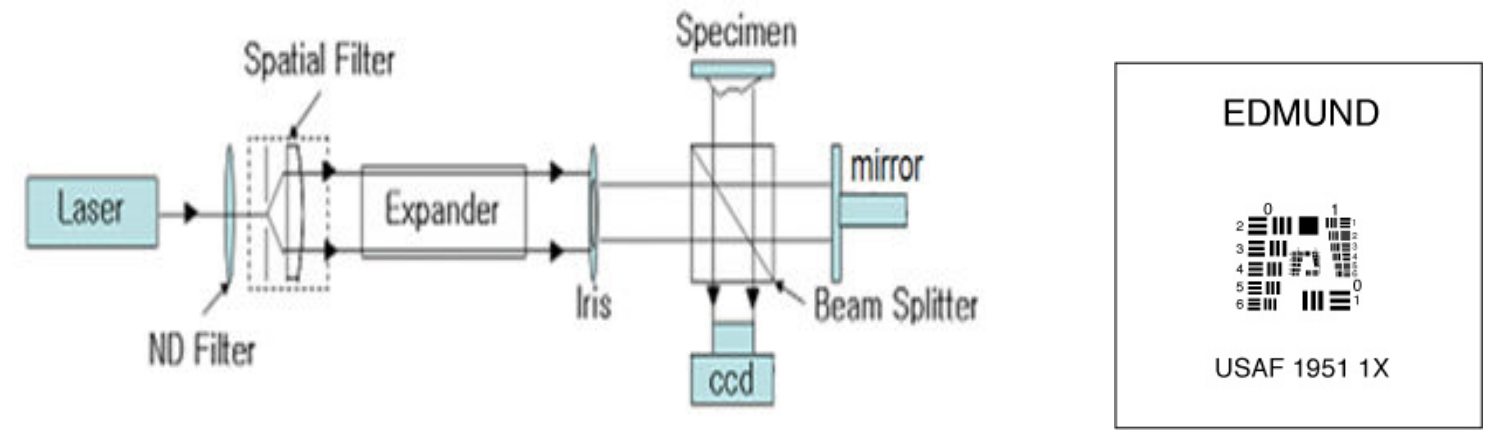

Fig.2.2 Michelson interferometer

Fig. 2.3 Shape of resolution target 


\subsubsection{Specimen}

Before accomplishing the experiment which sees the preliminary specimen resolution target for the performance appraisal of the interferometer used Edmund optics USAF 1951 models (positive and negative). Fig. 2.3 put out resolution target forms.

Respects a tensile experiment quality of material of the specimen which will be used the residual stress which occurs STS-304, the thickness 3mm Fig 2.4.(a) with uses a same machine work method and objective loss minimizes, the detail of the specimen comes Fig.2.4 (b).

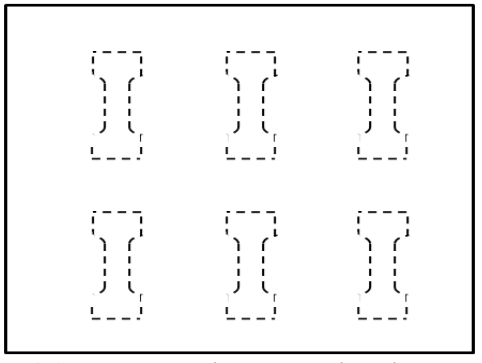

(a) Processing method



(b) Specimen specification

Fig. 2.4 Specimen Specifications (unit: $\mathrm{mm}$ )

\subsection{Experiment method}

Before accomplishing the experiment which tries to compose the interferometer measures resolution target experimented preliminarily. After preparation experiment, we measured rupture surface when become rupture with $3,5,7,9 \mathrm{~mm} / \mathrm{min}$ speed of tensile specimen. Also each specimen measurement area $(0 \mathrm{~mm}, 5 \mathrm{~mm}, 10 \mathrm{~mm}$ and $15 \mathrm{~mm}$ in the part rupture surface) measure surface roughness by using reflective interferometer.

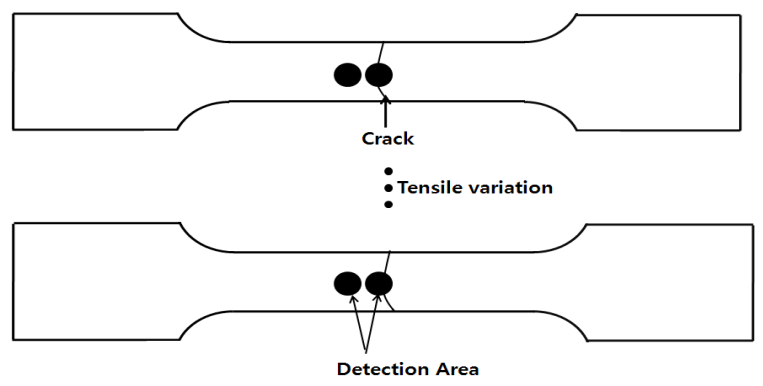

Fig.2.5 Sort of specimen detection zone

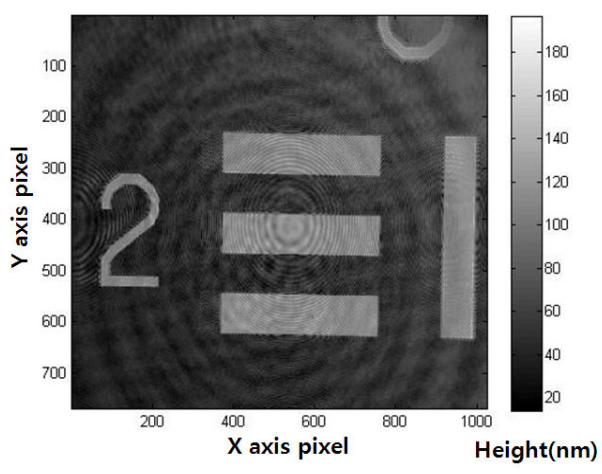

Fig.3.1 USAF1951 target hologram image $2 \mathrm{D}$ reconstruction

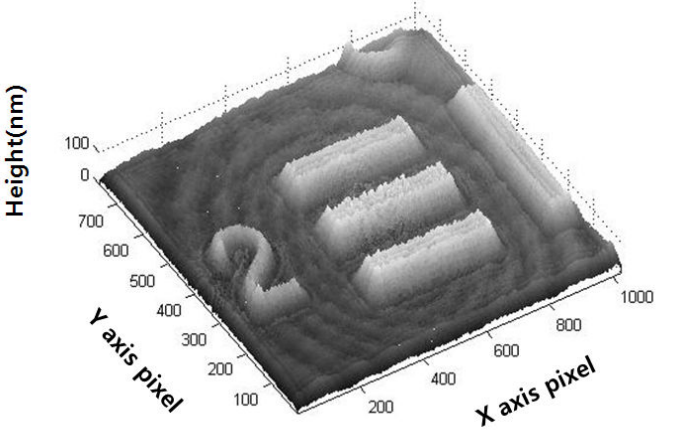

Fig.3.2 USAF1951 target hologram image $3 \mathrm{D}$ reconstruction 




Fig.3.3 Measurement height data processing

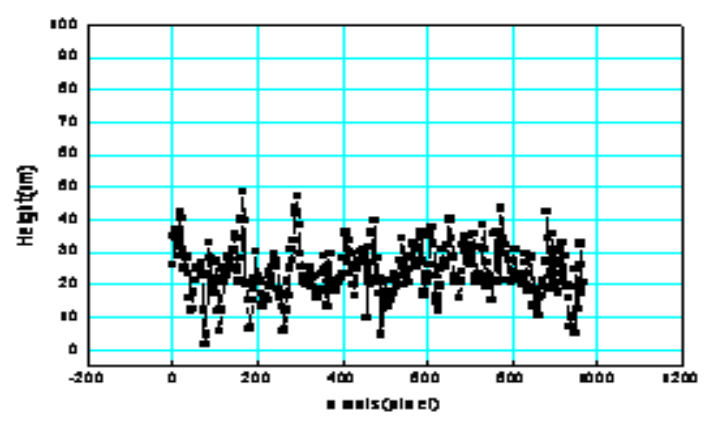

Fig. 3.4 Intensity distribution along Y pixel

\section{Results}

\subsection{Resolution target experiment}

The measurement object is Resolution (USAF1951). We got the hologram image by using Micro digital gage with focal length changed. Fig.3.1 show reconstructed image that use the image of 1 and $20 \mathrm{~cm}$ which distance between of CCD plane and surface plane. Fig 4-2 show three-dimension reconstructive image.

\section{2 surface roughness measurement method of machine work specimen}

We measured surface roughness of standard tensile specimen before each location surface roughness by tensile speed measured. Distance $10 \mathrm{~cm}$ between CCD plane and surface and the center department of the specimen surface roughness measured by using reflected interferometer of Fig 3-2. Fig.3.5 show two-dimension result and Fig.3.6 show reconstructed 3D surface of standard specimen. The graphs of Fig.3.4 express the surface of the standard specimen to follow Y-pixel. Experimental results are shown in the figures (3.3-6) analyzes we measure each part surface roughness by tensile speed.

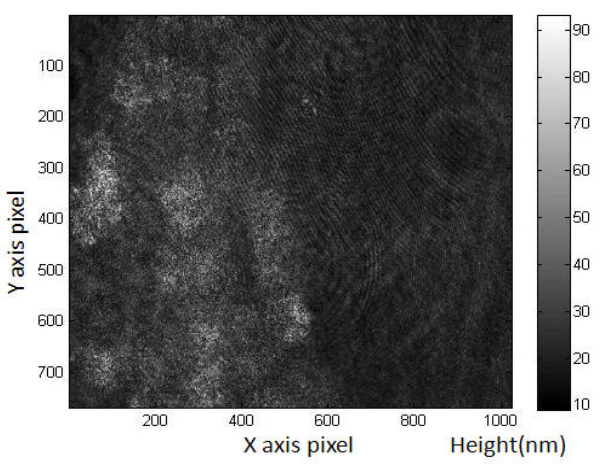

Fig.3.5 A surface of standard specimen 2 dimensional reconstruction

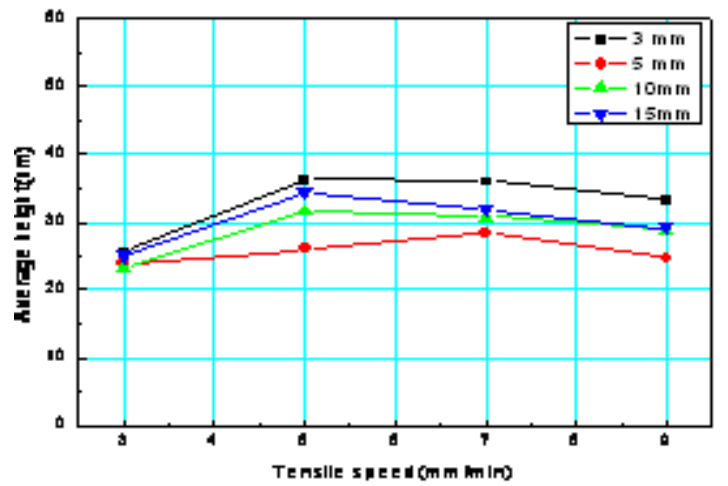

Fig.3.7 Comparison of average height by Fracture surface to distance

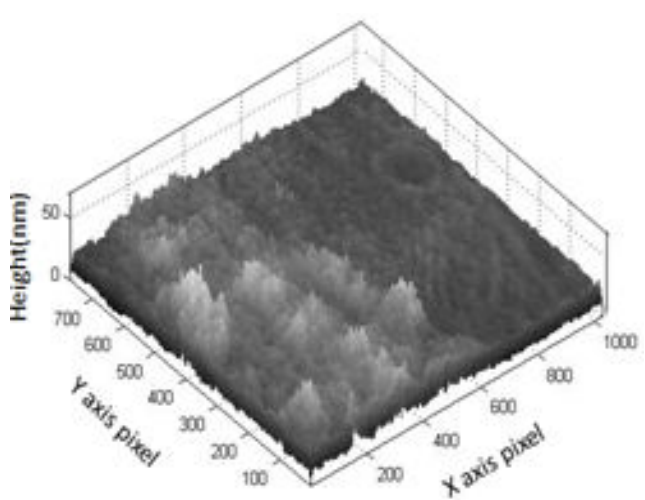

Fig.3.6 A surface of standard specimen 3 dimensional reconstruction

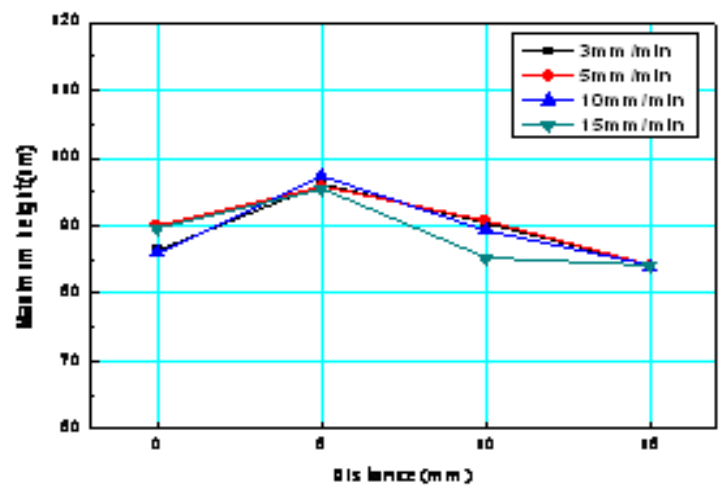

Fig.3.8 Comparison of Maximum height by distance 


\subsection{Each location roughness measurement according to tensile speed}

Each tensile speed rupture specimen measured surface roughness at rupture surface from $0 \mathrm{~mm}$, $5 \mathrm{~mm}, 10 \mathrm{~mm}$ and $15 \mathrm{~mm}$.

We measure height information of surface according to each reconstruct image. Fig.3.7, 3.8 shows measured result. The graph of Fig 3.7 see the average of surface roughness increased and decreases the form which was visible according to the distance will become more distant surface roughness from rupture plane. Like this result the local contraction actual condition occurs in rupture of the specimen, changed roughness undergoes the influence of actual condition of local contraction. Like this result be able to more clearly confirm from Fig 3.8.

\section{Conclusion}

In this paper, after making the standard specimen that is made of the material which is mostly used in mechanical structure, the phenomenon happening on the object in accordance with the external tensile condition was analyzed. Therefore, the changes that happened to the specimen were measured according to the external load conditions using digital holography and the conclusion of this paper is as follows.

1) The change of surface of the objects was measured until nano units with composing the micro digital holography.

2) The surface change measured constantly in accordance with the tensile speeds respectively using micro digital holography.

In this paper, the surface roughness by various tensile speeds were measured quantitatively, this could be utilized in the behavior analysis of mechanical structures.

\section{Acknowledgements}

This work was supported by Nuclear Research \& Development Program of National Research Foundation of Korea (NRF) funded by ministry of Education, Science \& Technology(MEST)

(Grant code: 2009-0082786)

\section{References}

[1]. D. Gabor, "A new microscopic principle," Nature. 1611 777-778

[2]. D. Gabor, "Microscopy by reconstructed wavefront," Proc. Roy. Soc. 197, 454-487(1949)

[3]. D. Gabor, "Microscopy by reconstructed wavefront II," Proc. Phys. Soc. 64, 449-469(1951)

[4]. J. W. Goodman, and R. W. Lawrence, "Digital phase formation from electronically detected holograms," Appl. Phys. Lett. 1, 77-79 (1967)

[5]. M. A. Kronrod, N. S. Merzlyakov and L. P. Yaroslavskii, "Reconstruction of a hologram with a computer," Sov. Phys. Tech. Phys. 17, 333-334 (1972)

[6]. U. Schnars, and W. Juptner, "Direct recording of hologram by a CCD target and numerical reconstruction," Appl. Opt. 33, 179-181 (1994)

[7]. B. Javidi, and E. Tajahuerce, "Three-dimensional object recognition by use of digital holography," Opt. Lett. 25, 610-612 (2000)

[8]. T. Zhang and I. Yamaguchi, "Three-dimensional microscopy with phse shifting digital holography," Opt. Lett. 23, 1221-1223 (1998)

[9]. B. C. Kim and S. W. Kim, "Absolute interferometer for three-dimensional profile 45 measurement of rough surfaces," Opt. Lett. 28, 528-530 (2003) 